\title{
COVID-19 reinfection in Beta-Thalassemia Major Patient: first
} case report.

\author{
Lina Okar $^{1}$, Rita Ahmad ${ }^{2}$, and mohamed yassin ${ }^{3}$ \\ ${ }^{1}$ Hamad Medical Corporation \\ ${ }^{2}$ Affiliation not available \\ ${ }^{3} \mathrm{HMC}$ NCCCR
}

November 6, 2020

\begin{abstract}
All people including those with Haemoglobinopathies are at risk of COVID-19 infection. Though those patients were considered vulnerable, a clear prognosis, as well as the possibility of reinfection, was not mentioned before. We report here the first case of Thalassemia beta major who had COVID-19 reinfection detected by screening.
\end{abstract}

\section{Introduction:}

Severe Acute Respiratory Syndrome -Coronavirus 2 (SARS-COV2) was responsible for the latest outbreak recently, presenting as mainly respiratory symptoms and carrying high mortality rate[1]. COVID-19 put all people at risk of being infected including haemoglobinopathies patients. Multiple factors among those patients increase their risk for infections yet the occurrence of reinfection and it is implication on them, and the community were not described before. Thalassemia is one of the most common haemoglobinopathies and requires life-long management and follow up. Splenectomy, regular blood transfusion and iron chelation therapy are essential components in the management plan. Unfortunately, they cause some complications on the long term and might raise the slurpability of current reinfection[2]. The impact of these factors on COVID-19 infection is not well known yet.

Here we present a case of 31-year-old splenectomized lady with transfusion dependent Beta Thalassemia Major had COVID-19 infection recurrence detected by screening. In this article we are discussing the possibility behind COVID-19 reinfection in thalassemia patients.

\section{Case presentation:}

A 31-year-old Pakistani lady with past medical history of transfusion dependent Beta Thalassemia major, type II diabetes Mellitus and hypothyroidism visited our hematology department for follow up and routine blood transfusion. Due to the ongoing spread of COVID-19 infection in Qatar, PCR via nasopharyngeal swap was done as part of the local screening plan before admission in day care for the blood transfusion and tested positive.

The patient was admitted before due to COVID-19 infection; her previous course was smooth without any complication as the current one. The period between the two positive PCR was 55 days. Her current management plan and home medications as follow: regular blood transfusion and iron chelation therapy for thalassemia, insulin, Sitagliptin/Metformin, levothyroxine and Tramadol. A comparison between the two presentation was mentioned below as well the investigations results. Table.1

\section{Discussion:}


Coronaviruses were known before for only their mild presenting symptoms like common cold. However, the world has recently seen a huge outbreak caused by Sever Acute Respiratory Syndrome Coronavirus (SARS-COV2) which was highly pathogenic and spread rapidly starting from China to all over the world with unfortunately increased mortality rate[3]. COVID-19 presentation was categorized into typical and atypical symptoms with fatigue, fever, dry cough and dyspnea being most common especially at onset of the infection, while headache, sore throat, runny nose, diarrhea and vomiting were considered as less common manifestations. However, asymptomatic cases were also documented and played an important role on the spread of the infection[4].

COVID-19 reinfection was reported before as some patients RT-PCR tested positive again after recovery. However, the mechanism is not clear and it is not known yet if affected patients might represent a carriers for the virus[5].

Hemoglobinopathies are well-known widely spread hematological disorders. Thalassemia is considered one of the most common form of those diseases, it is mainly due to reduction on either alpha or beta chains, this defect lead to wide range of clinical presentations. Both thalassemia Beta or Alpha type might also be categorized into transfusion dependent or independent. [6]

Severity of COVID-19 infection has been linked to many factors like the presence of other comorbidities such as diabetes, immunocompromised status, elderly and long term diseases like malignancy [7]. Date regarding patients with hemoglobinopathies vary in results. However the majority described a smooth course of infection and a favorable outcome. [8]

The current statement from Thalassemia International Federation (TIF) emphasize on the challenges during providing care for hemoglobinopathies patients in the epidemic, this concern arises mainly from the lack of clear evidence regarding COVID-19 infection progression, the need to visit health care facility frequently for follow up and blood transfusion[2].Thus, TIF also suggests that health care facility should take extra precaution while dealing with those patients. However, hemoglobin disorders has not been linked to a respiratory conditions in a direct way, yet close monitoring is necessary as thalassemia patients could have multi-organ damage including heart, lungs, liver, endocrine and immune system due to iron overload which might increase their COVID-19 complications risk[9-13].

Thalassemia patients are usually splenectomized as part of the therapeutic interventions provided for them, thus they are more vulnerable for recurrent bacterial infections. Till the day of writing this case there is no evidence that splenectomy is correlated with an increased risk of COVID-19 infection and complications[14].

Immune response to the pathogens differs among individuals due to genetic variations. Complications related to the impaired immunity in Beta Thalassemia patients were considered as an important cause of morbidity and mortality. Also, iron overload was labeled as the main reason behind immune deficiency in those patients. However, there is no evidence regarding iron chelation therapy role in COVID-19 patients with Beta Thalassemia major . [14]

To the best of our knowledge there is no reported cases in the literature for COVID-19 re-infection among hemoglobinopathies patients. It is worthy to notice that even with re-infection in our case the episode was smooth and detected with routine screening without even any respiratory symptoms during the whole quarantine period. However, the patient has multiple comorbidities, splenectomized, iron overload which might put her at increased risk for reinfection yet not for complications.

We suggest that even if Thalassemia patients seem to have a smooth clinical course of COVID-19 infection, the possibility of being re-infected should be kept on mind and screening them for COVID-19 infection should be done on each health care contact in order to provide close monitoring and avoid unexpected deterioration as the long-term out come is not known, as well due to the risk of spreading the infection especially if they are asymptomatic like this patient.

COVID-19 reinfection among Thalassemia patients is not reported before, so more research is needed to consider what pathology might make them vulnerable or at risk of reinfection. In our case we suggest that 
the presence of multiple comorbidities like diabetes, iron overload and splenectomy are possible reasons. Finally, we would like to raise a point that even in case of reoccurrence of COVID-19 infection in this thalassemia beta major patient the outcome still favorable, thus the recurrence of infection didn't mean poor prognosis or caused any complications but still important to screen them to control the spread of COVID-19.

\section{Conclusion:}

TIF considered patient with hemoglobinopathies in the vulnerable categories for COVID-19 complication and emphasized that those patients need extra precaution during their recurrent visits to the health care facilities. Mainly due to the lack of current evidence. However, published literature showed a favorable outcome on the majority of thalassemia patients who had COVID-19 infection. However, no data was found regarding the recurrence of this infection. We suggest that all physician should screen those patients for COVID-19 whenever the visited the hospital as they might develop asymptomatic recurrence and even though the clinical course seems to be smooth, screening is essential to control this pandemic and protect this vulnerable population as their outcome still unpredictable, thus offering them close monitoring and controlling their comorbidities.

\section{Acknowledgment}

We would like to acknowledge the Hematology section/ oncology department, Intensive care unit as well as Family medicine residency program at Hamad Medical Corporation for their support.

Open Access funding provided by the Qatar National Library

\section{Statement of Ethics}

Consent was obtained from the patients.

Case approved by HMC Medical Research center

\section{Declaration of interest}

The authors report no conflicts of interest.

\section{Authors contribution:}

All authors contributed equally to writing the manuscript.

\section{Data Availability Statement}

All data related to this article are available upon request.

\section{References:}

[1] Wang D, Hu B, Hu C, Zhu F, Liu X, Zhang J, et al. Clinical Characteristics of 138 Hospitalized Patients with 2019 Novel Coronavirus-Infected Pneumonia in Wuhan, China. JAMA - J Am Med Assoc 2020;323:1061-9. https://doi.org/10.1001/jama.2020.1585.

[2] de Sanctis V, Canatan D, Corrons JL, Karimi M, Daar S, Kattamis C, Soliman AT, Wali Y, Alkindi S, Huseynov V, Nasibova A. Preliminary Data on COVID-19 in patients with hemoglobinopathies: A multicentre ICET-A study. Mediterranean Journal of Hematology and Infectious Diseases. 2020;12(1).

[3] Song Z, Xu Y, Bao L, Zhang L, Yu P, Qu Y, et al. From SARS to MERS, thrusting coronaviruses into the spotlight. Viruses 2019;11. https://doi.org/10.3390/v11010059.

[4] Li H, Liu S, Yu X, Tang S, Tang C. Since January 2020 Elsevier has created a COVID-19 resource centre with free information in English and Mandarin on the novel coronavirus COVID- 19. The COVID-19 resource centre is hosted on Elsevier Connect, the company' s public news and information 2020. 
[5] He S, Zhou K, Hu M, Liu C, Xie L, Sun S, et al. Clinical characteristics of "re-positive" discharged COVID-19 pneumonia patients in Wuhan, China. Sci Rep 2020;10:17365. https://doi.org/10.1038/s41598020-74284-6.

[6] Okar L, Ali M, Parengal J, Yassin MA. COVID-19 and thalassemia beta major in splenectomized patient: Clinical case progression and literature review. Clin Case Reports 2020:1-5. https://doi.org/10.1002/ccr3.3345.

[7] Huang C, Wang Y, Li X, Ren L, Zhao J, Hu Y, et al. Clinical features of patients infected with 2019 novel coronavirus in Wuhan, China. Lancet 2020;395:497-506. https://doi.org/10.1016/S0140-6736(20)30183-5.

[8] De Sanctis V, Canatan D, Corrons JL, Karimi M, Daar S, Kattamis C, Soliman AT, Wali Y, Alkindi S, Huseynov V, Nasibova A. A comprehensive update of ICET-A Network on COVID-19 in thalassemias: what we know and where we stand. Acta bio-medica: Atenei Parmensis. 2020 Sep 7;91(3):e2020026-.

[9] Yassin MA, Soliman AT, De Sanctis V, Hussein RM, Al-Okka R, Kassem N, et al. Jadenu @ substituting Exjade (B) in iron overloaded $\beta$-thalassemia major (BTM) patients: A preliminary report of the effects on the tolerability, serum ferritin level, liver iron concentration and biochemical profiles. Mediterr J Hematol Infect Dis 2018;10. https://doi.org/10.4084/MJHID.2018.064.

[10] Soliman A, Yasin M, El-Awwa A, Osman M, de Sanctis V. Acute effects of blood transfusion on pituitary gonadal axis and sperm parameters in adolescents and young men with thalassemia major: a pilot study. Fertility and sterility. 2012 Sep 1;98(3):638-43..

[11] Soliman AT, Yassin M, Majuid NM, Sabt A, Abdulrahman MO, De Sanctis V. Cortisol response to low dose versus standard dose (back-to-back) adrenocorticotrophic stimulation tests in children and young adults with thalassemia major. Indian journal of endocrinology and metabolism. 2013 Nov;17(6):1046.

[12] Soliman AT, Prabhakaran Nair A, Al Masalamani MS, De Sanctis V, Abu Khattab MA, Alsaud AE, et al. Prevalence, clinical manifestations, and biochemical data of type 2 diabetes mellitus versus nondiabetic symptomatic patients with COVID-19: A comparative study. Acta Biomed 2020;91:e2020010. https://doi.org/10.23750/abm.v91i3.10214.

[13] Wang Y, Wang Y, Chen Y, Qin Q. Unique epidemiological and clinical features of the emerging 2019 novel coronavirus pneumonia (COVID-19) implicate special control measures. J Med Virol 2020;92:568-76. https://doi.org/10.1002/jmv.25748.

[14] Karimi M, De Sanctis V. Implications of SARSR-COV 2 infection in thalassemias: Do patients fall into the 'high clinical risk' category? Acta Biomed 2020;91:50-6. https://doi.org/10.23750/abm.v91i2.9592.

\section{List of figure and table legends:}

Table 1. comparison between the first presentation and the recurrence. 